\title{
A comprehensive phylogeny of birds (Aves) using targeted next-generation DNA sequencing
}

Richard O. Prum*, Jacob S. Berv, Alex Dornburg, Daniel J. Field, Jeffrey P. Townsend, Emily Moriarty Lemmon \& Alan R. Lemmon

\section{PAPER ABSTRACT}

Although reconstruction of the phylogeny of living birds has progressed tremendously in the last decade, the evolutionary history of Neoaves - a clade that encompasses nearly all living bird species - remains the greatest unresolved challenge in dinosaur systematics. Here we investigate avian phylogeny with an unprecedented scale of data: $>390,000$ bases of genomic sequence data from each of 198 species of living birds, representing all major avian lineages, and two crocodilian outgroups. Sequence data were collected using anchored hybrid enrichment, yielding 259 nuclear loci with an average length of 1,523 bases for a total data set of over $7.8 \times 10^{7}$ bases. Bayesian and maximum likelihood analyses yielded highly supported and nearly identical phylogenetic trees for all major avian lineages. Five major clades form successive sister groups to the rest of Neoaves: (1) a clade including nightjars, other caprimulgiforms, swifts, and hummingbirds; (2) a clade uniting cuckoos, bustards, and turacos with pigeons, mesites, and sandgrouse; (3) cranes and their relatives; (4) a comprehensive waterbird clade, including all diving, wading, and shorebirds; and (5) a comprehensive landbird clade with the enigmatic hoatzin (Opisthocomus hoazin) as the sister group to the rest. Neither of the two main, recently proposed Neoavian clades-Columbea and Passerea-were supported as monophyletic. The results of our divergence time analyses are congruent with the palaeontological record, supporting a major radiation of crown birds in the wake of the Cretaceous-Palaeogene (K-Pg) mass extinction.
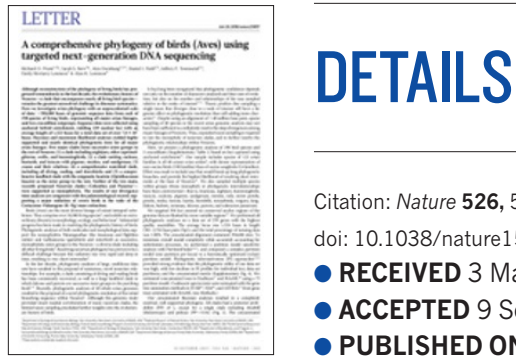

\author{
Citation: Nature 526, 569-573 (2015) \\ doi: 10.1038/nature 15697 \\ - RECEIVED 3 May 2015 \\ - ACCEPTED 9 September 2015 \\ - PUBLISHED ONLINE 7 October 2015
}

\section{SUMMARY}

The backbone and timescale of the avian tree of life. It has been difficult to get a clear picture of how and when birds evolved into the huge variety of form and function that we see today. A new phylogenetic analysis of genes from 198 living bird species and two crocodilians helps to resolve the view.

\section{The problem}

Birds are among the most diverse and ubiquitously distributed groups of tetrapods. More than 10,000 living species of bird have been recognized so far ${ }^{1}$, and they occupy a great diversity of ecosystems. They exhibit a dizzying variety of ecologies, morphologies, colours, life histories and breeding systems, spanning everything from the diurnal, hovering, nectar-feeding hummingbird to the nocturnal, flightless, worm-eating kiwi.

Unravelling how, when and why this spectacular diversity evolved demands an accurate hypothesis of the evolutionary interrelationships among the major groups of living birds. But despite more than a century of interest in avian evolution - and efforts based on skeletal morphology $^{2}$, genetic-distance methods ${ }^{3}$ and, more recently, DNA sequences ${ }^{4}$ - a clear picture of the avian tree of life has been frustratingly elusive.

Why has this issue proven so difficult to resolve? Palaeontological data suggest that, around 66 million years ago, the Cretaceous-Palaeogene mass extinction event nearly wiped out the antecedents of living birds ${ }^{5}$. Precisely dating the subsequent diversification (or 'radiation') of birds has proven highly controversial ${ }^{6}$, but the fossil record suggests that most of the divergence might have taken place within a narrow temporal window (about ten million years) in the wake of that mass extinction. This remarkably rapid radiation has probably obscured many of the evolutionary relationships among the major groups of living birds.

\section{The solution}

Overcoming the challenge of resolving these historically controversial relationships demanded improvements in gene-sequencing technology and associated analytics. By using a technique known as targeted 
anchored enrichment ${ }^{7}$, we generated an unprecedented data set comprising 259 genetic loci, filtered for maximal phylogenetic information $^{8}$, from 198 bird species (representing virtually every major group). We inferred both the relationships among these major groups and a timescale for their radiation. Together, these results provide the clearest picture yet of the bird tree of life, and give an idea of when in Earth's history the major evolutionary divergences among birds took place.

\section{The interpretation}

We discovered that living birds are divided into seven major groups that sprang forth rapidly in the wake of the Cretaceous-Palaeogene mass extinction (diverging from one another within some 15 million years of this event). We also found that modern bird groups are considerably younger than previously thought. The most recent common ancestor of all living birds lived about 72 million years ago, with the ancestors of as few as three modern lineages of flying birds surviving the extinction event that wiped out the non-avian dinosaurs.

Our analyses revealed some astonishing conclusions. For example, nearly all aquatic bird lineages (excluding ducks, rails, kingfishers and dippers) share just one common ancestor, suggesting that birds have adapted to an aquatic lifestyle much less frequently than previously assumed. Also, our results provide strong support for the hypothesis that the ancestor of the modern 'landbird' radiation - including woodpeckers, parrots and song birds - was some kind of raptor.

We also have a new solution to one of the world's great phylogenetic mysteries. The strange morphology and leaf-eating behaviour of the hoatzin (Opisthocomus hoazin, a strange Amazonian species) have shrouded its evolutionary relationships for decades. We show that the hoatzin sits on the longest solitary branch in the entire avian tree of life, last sharing a common ancestor with another living species nearly 66 million years ago.

By clarifying the backbone of the avian tree of life, our research helps to resolve one of the longest-standing mysteries in vertebrate systematics. It sets the stage for future analyses as we move towards assembling a comprehensive picture of the avian tree of life that incorporates all 10,000 living species. .

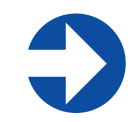

The full version of this article is at Nature's website at go.nature.com/29kkyu2

\section{GRAPHICAL ABSTRACT}

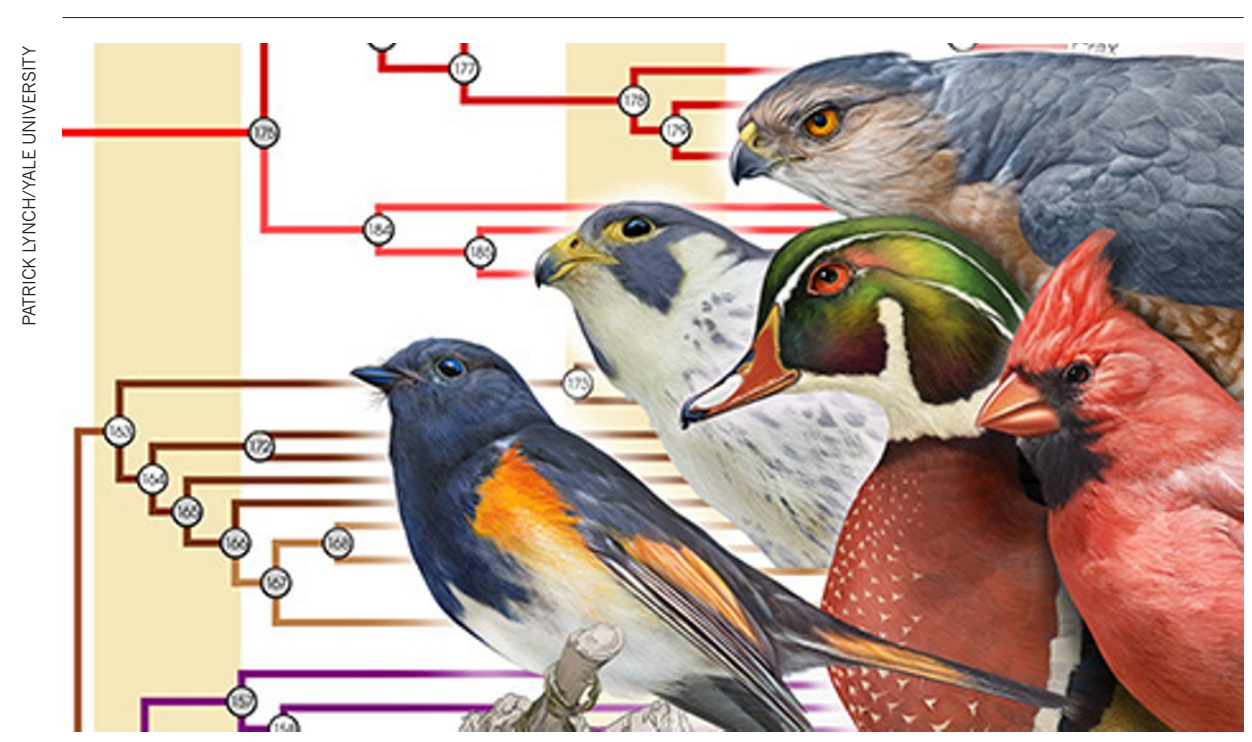

The avian tree of life. By using a next-generation technique known as targeted genetic enrichment, we isolated 259 genetic loci from 198 living bird species and two crocodilians, and constructed a phylogeny. The tree reveals a clear picture of avian evolution. It shows, for example, that most of the major bird groups appeared within about 15 million years of the Cretaceous-Palaeogene mass extinction, and that most water birds are closely related.

\section{FURTHER READING}

1. Gill, F. B. Ornithology 2nd edn (W. H. Freeman, 1995).

This is a general reference work on the diversity and biology of birds.

2. Livezey, B. C. \& Zusi, R. L. Higher-order phylogeny of modern birds (Theropoda, Aves: Neornithes) based on comparative anatomy. II. Analysis and discussion. Zool. J. Linn. Soc. 149, 1-95 (2007).

A comprehensive phylogenetic hypothesis for living birds based on morphology.

3. Sibley, C. G. \& Ahlquist, J. E. Phylogeny and Classification of Birds: A Study in Molecular Evolution (Yale Univ. Press, New Haven, 1990). Comprehensive analysis of the relationships of more than 1,000 bird species based on DNA-DNA hybridization, a genetic distance method.

4. Hackett, S. J. et al. A phylogenomic study of birds reveals their evolutionary history. Science 320, 1763-1768 (2008). A pioneering phylogenomic study that discovered several clades confirmed by this study, including the sister-group relationship between parrots and perching birds.

5. Longrich, N. R., Tokaryk, T. \& Field, D. J Mass extinction of birds at the CretaceousPaleogene (K-Pg) boundary. Proc. Natl Acad. Sci. USA 108, 15253-15257 (2011). An analysis of the K-Pg extinction of North American birds.

6. Cracraft, J. et al. Response to Comment on "Whole-genome analyses resolve early branches in the tree of life of modern birds". Science 349, 1460 (2015).

A recent discussion of the age of the radiation of living birds.

7. Lemmon, A. R., Emme, S. A. \& Lemmon, E. M. Anchored hybrid enrichment for massively high-throughput phylogenomics. Syst. Biol. 61, 727-744 (2012).

Description of the anchored enrichment technique used to obtain the data in this analysis.

8. Townsend, J. P. Profiling phylogenetic informativeness. Syst. Biol. 56, 222-231 (2007).

Original paper describing the metric and methods for evaluating the phylogenetic informativeness of genetic loci that was used in this analysis.

\section{RELATED CONTENT}

Whole-genome analyses resolve early branches in the tree of life of modern birds

E. D. Jarvis et al.

go.nature.com/29npudf

An avian explosion

G. H. Thomas

go.nature.com/2a472kh 